\title{
Sind Kryptowährungen wie Bitcoin (BTC), Ethereum (ETH) und Ripple (XRP) als Sachen im Sinne des ZGB zu behandeln?
}

Benedikt Seiler / Daniel Seiler *

Die Autoren gehen der Frage nach, ob Kryptowährungen als Sachen im Sinne des ZGB aufgefasst und daran namentlich Eigentumsrechte begründet werden können. Dabei werden zunächst technische Grundlagen der sog. Blockchain-Technologie und der drei nach Markkapitalisierung grössten Kryptowährungen Bitcoin (BTC), Ethereum (Ether, ETH) und Ripple (XRP) dargestellt. Sodann wird untersucht, ob diese Kryptowährungen die Eigenschaften erfüllen, welche für die Qualifikation eines Objektes als Sache im Sinne des ZGB vorliegen müssen, und welches die Folgen einer solchen Qualifikation sind. Im Sinne einer modernen Auslegung des Sachbegriffs schliessen sich die Autoren der Auffassung an, wonach Kryptowährungen grundsätzlich als Sache und damit als Gegenstand des Eigentums zu behandeln sind.

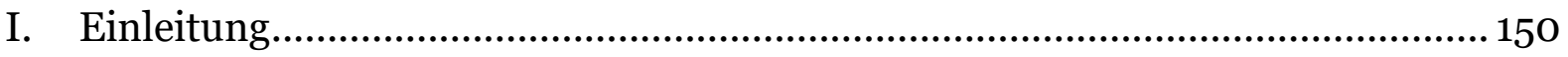

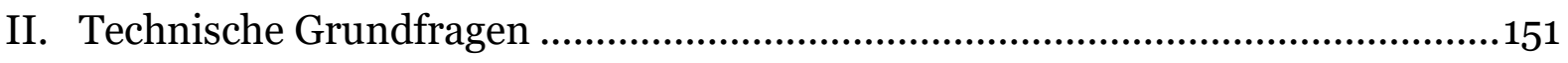

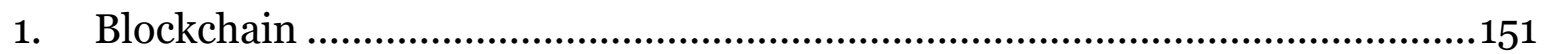

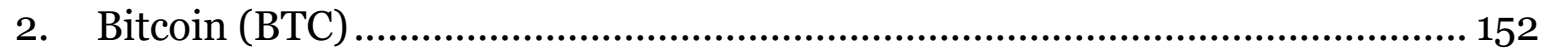

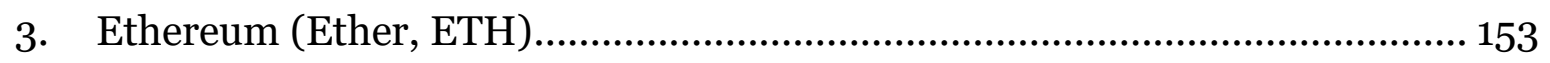

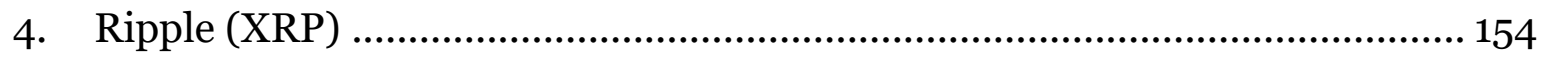

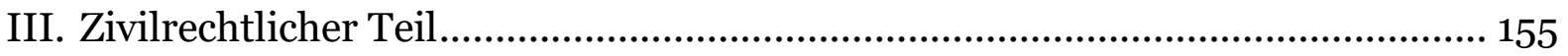

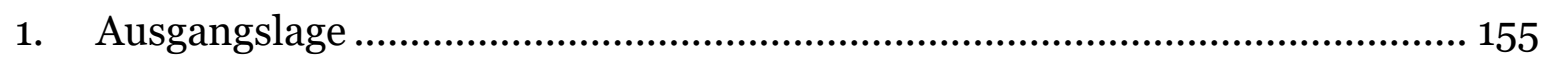

2. Der Sachbegriff des ZGB als dynamischer Begriff.................................... 156

3. Kryptowährungen als «Sache» im Sinne des ZGB? ................................. 156

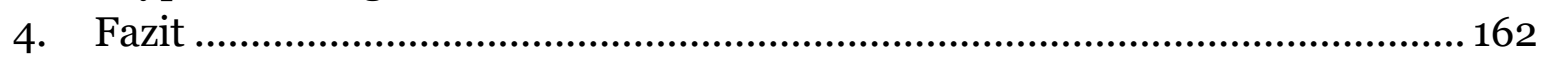

Zitiervorschlag: Benedikt Seiler/Daniel Seiler, Sind Kryptowährungen wie Bitcoin (BTC), Ethereum (ETH), Ripple (XRP) und Co. als Sachen im Sinne des ZGB zu behandeln?, in: sui-generis 2018, S. 149

URL: $\quad$ sui-generis.ch/65

DOI: $\quad$ https://doi.org/10.21257/sg.65

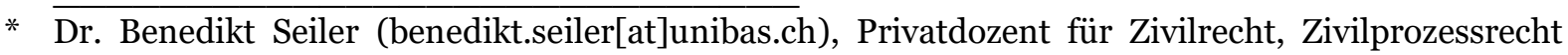
sowie Schuldbetreibungs- und Konkursrecht an der Universität Basel, Gerichtsschreiber am Appellationsgericht Basel-Stadt; Daniel Seiler (dseiler[at]kpmg.com), Senior Manager, Legal, KPMG Schweiz, spezialisiert auf digitales Recht, IT-Recht, Compliance, Datenschutz und Legal Transformation.

Dieses Werk ist lizenziert unter einer Creative Commons Namensnennung - Weitergabe unter gleichen Bedingungen 4.0 International Lizenz. 


\section{Einleitung}

1 Die bekannteste Kryptowährung Bitcoin existiert seit dem Jahr 2009 und blieb zunächst einige Jahre praktisch unbekannt. Noch im Jahr 2014 bezeichnete der Bundesrat Kryptowährungen als «Randphänomen» und kam deswegen zum Schluss, dass kein gesetzgeberischer Handlungsbedarf bestehe. ${ }^{1}$ Die wirtschaftliche Bedeutung und Marktkapitalisierung von Kryptowährungen stieg seither jedoch rasant. Der Durchbruch erfolgte im Jahr 2017, im Verlaufe dessen die globale Marktkapitalisierung von Bitcoin und anderen Kryptowährungen (sog. Altcoins) wie Ethereum (ETH), Ripple (XRP) usw. insgesamt auf einen Gegenwert von über 800 Milliarden Dollar anstieg. ${ }^{2}$ Aufgrund dieser Entwicklung schloss Christine Lagarde, Direktorin des Internationalen Währungsfonds, im September 2017 selbst eine komplette Ablösung der nationalen Währungen durch Kryptowährungen nicht mehr völlig aus. Auch der Bundesrat erkennt in Kryptowährungen nunmehr eine nachhaltige Entwicklung. Anlässlich der »Crypto Conference St. Moritz» im Januar 2018 bezeichnete Bundesrat Schneider-Amann Kryptowährungen als «Teil der vierten industriellen Revolution» und äusserte die Hoffnung, dass die Schweiz zur «KryptoNation» werde. 3

Aufgrund der wachsenden wirtschaftlichen und globalen Bedeutung von Kryptowährungen und der damit einher-

1 Bericht des Bundesrats vom 25. Juni 2014 in Beantwortung der Postulate Schwaab (13.3678) und Weibel (13.4070).

2 Die drei genannten Kryptowährungen BTC, ETH und XRP weisen derzeit die grösste Marktkapitalisierung auf (www.coinmarketcap.com)

3 Vgl. etwa SRF, Tagesschau vom 18. Januar 2018. gehenden immer lauter werdenden Forderung nach Regulierung zeichnete sich das erste Quartal 2018 durch starke Verunsicherungen am Markt aus. Dies auch deshalb, weil unterschiedliche Nationen eine zum Teil völlig unterschiedliche Position gegenüber Kryptowährungen einnehmen.4 In dieser Phase haben sich zwei der weltweit grössten Kryptowährungsplattformen binances ${ }^{5}$ und bitfinex ${ }^{6}$ zu einer Verlegung ihrer Tätigkeit nach Europa entschlossen. Letztere wird voraussichtlich als AG in der Schweiz angesiedelt.7 Damit ist die Bedeutung der Schweiz als Land im Krypto-Ökosystem potentiell erneut gestiegen. Weiter akzentuiert hat sich somit auch die Dringlichkeit einer «Implementierung» von Kryptowährungen in das Schweizer Recht, vor allem, wenn man sich vor Augen führt, dass bitfinex knapp zwei Millionen registrierte Nutzer aufweist und im bislang stärksten Monat Dezember 2017 nach eigenen Angaben einen Umsatz von über 100 Milliarden Dollar generierte. ${ }^{8}$

3 Kryptowährungen stellen den Gesetzgeber jedoch vor eine vielfältige Herausforderung, namentlich auf den Gebieten der Geldwäschereigesetzgebung, des Steuerrechts, des Strafrechts, des Privatrechts sowie des Vollstreckungsrechts, zumal selbst grundlegendste Rechtsfragen noch

$4 \longdiv { \text { Für eine Übersicht über die Positionen verschie- } }$ dener Nationen, vgl. Law \& Trust International, Crypto-Friendly Countries.

www.binance.com. www.bitfinex.com.

7 Marc Badertscher, Bitcoin-Börse Bitfinex will in die Schweiz ziehen, Handelszeitung, 27. März 2018.

8 www.bfxdata.com. 
weitgehend ungeklärt sind. 9 Aus zivilrechtlicher Sicht stellt sich insbesondere die Frage, ob Kryptowährungen als Sache im Sinne des ZGB behandelt werden können (nachfolgend Teil III). Hierfür ist es erforderlich, sich vorweg mit einigen wichtigen Grundlagen der technischen Eigenschaften und Funktionsweise von Kryptowährungen vertraut zu machen, soweit sie für die anschliessende rechtliche Würdigung von Bedeutung sind (nachfolgend Teil II).

\section{Technische Grundfragen}

\section{Blockchain}

4 Bei einer sog. Blockchain ${ }^{10}$ (Blockkette) handelt es sich um eine kontinuierlich erweiterbare Liste von Datensätzen. Diese als «Blöcke» bezeichneten Datensätze sind mittels kryptographischer Verfahren miteinander verkettet. Jeder einzelne Block enthält dabei stets einen kryptographisch sicheren Streuwert (Hash) des vorhergehenden Blocks sowie einen Zeitstempel und die Transaktionsdaten. ${ }^{11}$

5 Das der Blockchain zugrunde liegende Prinzip ist an sich nicht neu. So nimmt der Notar, der einen Grundstückkauf beurkundet, Bezug auf ein Dokument,

9 Stellungnahme des Bundesrats vom 31. Januar 2018 in Beantwortung der Interpellation Schmid (17.4024).

10 Economist Staff, Blockchains: The great chain of being sure about things, The Economist. 31. Oktober 2015; Nathaniel Poppe, A Venture Fund With Plenty of Virtual Capital, but No Capitalist, New York Times, 21. Mai 2016.

11 Arvind Narayanan/Joseph Bonneau/Edward Felten/Andrew Miller/Steven Goldfeder, Bitcoin and cryptocurrency technologies: a comprehensive introduction, Princeton 2016; John Pavlus, Die Welt des Bitcoin, Spektrum der Wissenschaft 4/2018, S. 15 . dass dem Verkäufer sein bisheriges Eigentum bescheinigt, und fügt der Kette derartiger Dokumente ein neues Glied hinzu. Dieses bezieht seine Gültigkeit aus der Verknüpfung mit dem Vorgängerdokument. Neu ist die Übertragung des Konzepts auf ein manipulationssicheres digitales System.12 Die Blockchain ist somit nicht eine in sich spezifische Technologie, sondern vielmehr ein Konzept für ein dezentrales Buchführungssystem, mithilfe dessen Konsens über den richtigen Zustand der Buchführung erzielt wird, selbst wenn (oder gerade weil) viele Teilnehmer an der Buchführung beteiligt sind - dieses Konzept wird auch als Distributed-Ledger-Technologie (DLT) bezeichnet. ${ }^{13}$

6 Es ist für den Begriff Blockchain unerheblich, worüber in derartigen Buchführungssystemen letztlich Buch geführt wird. Entscheidend ist vielmehr das Prinzip, dass die späteren Transaktionen auf den früheren Transaktionen aufbauen und sich die späteren Transaktionen dadurch als richtig bestätigen, indem sie die Kenntnis der früheren Transaktionen beweisen. Durch diese Vorgehensweise wird es systembedingt verunmöglicht, die Existenz oder den Inhalt der jeweils früheren Transaktionen zu manipulieren oder zu löschen, ohne dabei gleichzeitig alle späteren Transaktionen ebenfalls zu zerstören. Die anderen Teilnehmer der dezentralen Buchführung (sog. Nodes) haben aufgrund der Verteilung der Buch-

12 Alexander Lipton/Alex Pentland, Ausweg aus dem Bankenmonopol, Spektrum der Wissenschaft 4/2018, S. 23.

13 Blockchain \#Banking: ein Leitfaden zum Ansatz des Distributed Ledger und Anwendungsszenarien, in: Bundesverband Informationswirtschaft (Hrsg.), Telekommunikation und neue Medien, Berlin 2016. 
führung auf alle Teilnehmer Kenntnis dieser späteren Transaktionen und werden eine manipulierte Kopie der Blockchain daran erkennen, dass diese Abweichungen in den Berechnungen aufweist. Eine Verifizierung der manipulierten Transaktion erscheint somit als ausgeschlossen. ${ }^{14}$

7 Das Verfahren der Blockchain, also die kryptographische Verkettung in einem dezentralen Buchführungssystem bzw. einer Transaktionsdatenbank ist die technische Voraussetzung für Kryptowährungen und liegt diesen zugrunde. Gegenüber zentralen Systemen bietet das verteilte System eine Verbesserung der Transaktionssicherheit und eine Vereinfachung der einzelnen Transaktion. Eine Blockchain ermöglicht es insbesondere, dass in einem dezentralen Netzwerk eine Einigkeit zwischen den Teilnehmern (Nodes) erzielt werden kann, obwohl sich die Teilnehmer weder kennen noch deren Identität bekannt ist. Beim verteilten Transaktionssystem, das für die Kryptowährung Bitcoin genutzt wird, handelt es sich um die älteste sich in Betrieb befindliche Blockchain und damit den ersten, breiten Anwendungsfall derselben. ${ }^{15}$

\section{Bitcoin (BTC)}

8 Bitcoin (BTC) bezeichnet eine Währung, welche auf einem digitalen, kryptographischen System basiert (sog. Krypto-

$14 \overline{\text { Luzius Meissner, Kryptowährungen: Geschichte, }}$ Funktionsweise, Potential, in: Weber/Thouvenin (Hrsg.), Rechtliche Herausforderungen durch webbasierte und mobile Zahlungssysteme, Zürich 2015, S. 84; Pavlus (Fn. 11), S. 15 f.

15 Pavlus (Fn. 11), S. 15 f.; vgl. Meissner (Fn. 14), S. $76 \mathrm{f}$. währung). ${ }^{16}$ Erstmals wurde das BitcoinZahlungssystem im Jahr 2008 in einem Dokument beschrieben, welches unter dem Pseudonym Satoshi Nakamoto veröffentlicht wurde. ${ }^{17}$ Kurz darauf wurde eine erste Software veröffentlicht (ein sog. Bitcoin-Client). ${ }^{18}$ Der Wert eines Bitcoins bestimmt sich allein durch Angebot und Nachfrage..$^{19}$

9 Um am Bitcoin-Zahlungssystem teilzunehmen, ist es lediglich notwendig, einen Bitcoin-Client oder einen entsprechenden Online-Dienst zu nutzen. Diese Eigenschaft erlaubt es, dass das BitcoinSystem keiner geographischen Beschränkung unterliegt, da einzig ein Client und eine Internetverbindung notwendig sind. Der Einsatz ist somit länderübergreifend möglich. ${ }^{20}$ Eine der zentralen Eigenschaften des Bitcoin-Systems und der zugrundeliegenden Blockchain ist der Umstand, dass mittels Kryptographie garantiert werden kann, dass Transaktionen mit Bitcoins nur vom jeweiligen Inhaber vorgenommen werden können und dass es zudem unmöglich ist, Geldeinheiten mehrfach auszugeben. ${ }^{21}$

16 Money and Capital, in: Brian Duignan (Hrsg.), Economics: Taking the Mystery Out of Money Series, New York 2012, S. 48.

17 Joshua Davis, The Crypto-Currency: Bitcoin and its mysterious inventor, The New Yorker, 10. Oktober 2011.

18 «To reduce confusion between Bitcoin-thenetwork and Bitcoin-the-software we have renamed the reference client to Bitcoin Core.» Bitcoin Core version 0.9.0 released -Rebranding to Bitcoin Core, Bitcoin Project, 19. März 2014; Usman Chohan, Cryptocurrencies: A Brief Thematic Review, Social Science Research Network (SSRN), 4. August 2017.

19 Usman Chohan, A History of Bitcoin, Social Science Research Network (SSRN), 30. September 2017.

20 Wikipedia, Bitcoin, 6. Mai 2018.

21 Axel Kannenberg, Kryptogeld-Regulierung in Deutschland: «Bitcoins fair behandeln», heise online, 27. September 2014. 
Soll mit Bitcoins gehandelt werden, ist es zunächst erforderlich, eine entsprechende Software (bei PCs) oder eine entsprechende mobile Applikation (bei Smartphones) zu installieren. Diese verbindet sich via Internet über ein dezentrales Netzwerk (sog. Peer-to-Peer Netzwerk) und lädt die Blockchain-Datenbank herunter; man wird so zu einem Teil des Blockchain-Netzwerks und dem damit verbundenen Betrieb des BitcoinProtokolls. ${ }^{22}$ Der Ablauf einer Transaktion von Bitcoins ist zwar je nach genutzter Software im Detail anders ausgestaltet, der zugrundeliegende Vorgang ist aber immer derselbe: Es wird einerseits der eigene private Schlüssel (sog. private key) benötigt, zudem der aus der Zieladresse abgeleitete öffentliche Schlüssel des Empfängers (sog. public key). Letzterer hat die Funktion, die Transaktion derart mit dem eigenen private key zu signieren, dass der Empfänger die nächste Transaktion wiederum nur mit seinem eigenen private key gültig signieren kann. ${ }^{23}$ An dieser Ausgestaltung der Transaktion ist der Mechanismus der Blockchain deutlich zu sehen: Es findet kein (wechselseitiger) Austausch oder eine Übergabe von Daten oder Gegenständen statt, vielmehr wird eine neue Signatur generiert, welche einer bestehenden Kette von vergangenen Signaturen der Blockchain hinzugefügt wird. ${ }^{24}$ Diese Verkettung der vergange-

$2 2 \longdiv { \text { Eine eindrückliche geographische Übersicht über } }$ alle $\mathrm{zu}$ einem gegebenen Zeitpunkt mit dem Netzwerk verbundenen Computer findet sich unter Bitnodes.

23 Stephan D. Meyer/Benedikt Schuppli, «Smart Contracts» und deren Einordnung in das schweizerische Vertragsrecht, recht 2017, S. 219, 204 ff., 205.

24 Nicolas Jacquemart/Stephan D. Meyer, Der Bitcoin-/Bitcoin-Cash-Hardfork, GesKR 2017, S. 469 nen Signatur mit einer neuen Signatur auf der Blockchain macht diese einzelne Transaktion $\mathrm{zu}$ einem einmaligen, irreversiblen und für alle Nutzer der Blockchain offenen und transparent nachvollziehbarem Ereignis, welches nicht in gleicher Art ein zweites Mal auftreten kann, womit auch eine Mehrfachtransaktion (sog. double spend) oder eine Kopie bzw. eine Multiplikation von Bitcoins technisch ausgeschlossen ist.

11 Wie beschrieben ist für den Zugriff bzw. für die Verfügung über Kryptowährungen wie z.B. Bitcoin jeweils ein kryptographisches Schlüsselpaar erforderlich. Bei den kryptographischen Schlüsseln handelt es sich um eine Bitfolge, ${ }^{25}$ also eine digitale Zeichenfolge, welche mittels Zahlen und Buchstaben oder beispielsweise auch mittels eines sog. QR-Code dargestellt werden kann. Diese Daten werden von den Nutzern meist in einer Software gespeichert, einem sogenannten Wallet. Die Wallet-Software ermöglicht die Aufbewahrung und Verwaltung der Schlüssel sowie die Anzeige der Kryptowährungseinheiten ${ }^{26}$ auf dem PC oder dem Smartphone. Es ist auch möglich, wenn auch im Umgang wenig komfortabel, die eigenen kryptographischen Schlüssel beispielsweise auf Papier aufzuschreiben bzw. auszudrucken und auf diese Weise aufzubewahren.

\section{Ethereum (Ether, ETH)}

12 Bei Ethereum handelt es sich ebenfalls um ein verteiltes System. Dieses ist besonders dafür ausgelegt, dezentrale

\footnotetext{
25 Das Wort Bit ist eine Wortkreuzung aus binary digit - englisch für «binäre Ziffer» oder auch «Binärziffer», vgl. Wikipedia, Bit, 3. Mai 2018.

26 Jacquemart/Meyer (Fn. 24), S. 469 f.
} 
Verträge (sog. Smart Contracts) ${ }^{27}$ und Programme mittels einer eigenen Blockchain anzulegen, zu verwalten und allenfalls auszuführen. ${ }^{28}$ Das System Ethereum verwendet zur Vergütung der Transaktionsverarbeitungen die interne Kryptowährung Ether (ETH) als Zahlungsmittel für die Teilnehmer (Nodes). Im Unterschied zu Bitcoin ist Ethereum jedoch keine reine Kryptowährung, sondern eine Plattform für sogenannte Dapps (Distributed Apps), die aus Smart Contracts bestehen. Für Smart Contracts gibt es eine Vielzahl von Anwendungen, unter anderem E-Voting-Systeme, virtuelle Organisationen, Identitätsmanagement und Crowdfunding. ${ }^{29}$

13 Aufgrund der Tatsache, dass Ethereum bzw. Ether (ETH) Blockchain als Technologiegrundlage einsetzt, sind auch die via Ethereum bzw. Ether verarbeiteten Transaktionen gleichsam unwiderruflich bzw. unveränderbar. Auch hier lässt sich die Transaktionskette beliebig weit zurückverfolgen, womit die Transparenz und Nachvollziehbarkeit der Transaktionen gewährleistet ist. 30

\section{Ripple (XRP)}

14 Das Ripple-System basiert auf einer verteilten, öffentlichen Datenbank, welche ein Register mit allen Kontoständen enthält. Dieses Register kann jeder einsehen. Eine Besonderheit des RippleSystems ist die Möglichkeit, dass es zusätzlich zu den Kontoständen auch Informationen über Kaufs- und Verkaufsangebote von Devisen und Gütern

27 Eingehend hierzu Meyer/Schuppli (Fn. 23), S. $204 \mathrm{ff}$.

28 Ethereum Blockchain, Blockchain Nachrichten, 15. November 2016.

29 www.ethereum.org.

30 www.ethereum.org. enthalten kann, wodurch es zu einem Handelsplatz wird. Auch beim RippleSystem einigen sich die Teilnehmer auf Änderungen im Register nach einem Konsensverfahren. ${ }^{31}$ Zusätzlich zum Zahlungsnetzwerk und dem Handelsplatz enthält das Ripple-System eine eigene interne Kryptowährung XRP32, welche unwiderrufliche bzw. unveränderbare und transparente Transaktionen ermöglicht. 33

15 Im erwähnten Register aller Kontostände ist durch Schuldscheine, auch genannt IOUs (für «I owe you») erfasst, wer wie viel von welcher Währung einem Teilnehmer schuldet und umgekehrt. Wichtig in diesem System sind insbesondere auch die sog. Ripple-Gateways, denn diese nehmen etablierte Währungen wie z.B. Dollar an und stellen dafür IOUs aus, welche jederzeit wieder in eine Währung eingelöst werden können. 34 Im Unterschied zu anderen Kryptowährungen wie Bitcoin oder Ethereum (ETH) ist eine Geldschöpfung durch sog. Mining nicht vorgesehen.

16 Vorteile des Ripple-Protokolls sind der Umstand, dass es sich um einen offenen Standard handelt, der bestehende Zahlungsnetzwerke verbinden kann, dass die Nutzer in einer wechselseitigen Schuld stehen können, dass die Transaktionen sekundenschnell verarbeitet werden können und dass Ripple das Potential besitzt, als eine Art Übersetzer zwischen beliebigen anderen Währungen zu funk-

31 How Ripple Works (www.ripple.com).

32 Top 9 Frequently Asked Questions About Ripple and XRP, FAQ, Ripple.

33 FAQ, Ripple.

34 Wikipedia, Ripple (Geldsystem), 5. Mai 2018. 
tionieren.35 Dass dieses Potential nicht bloss theoretischer Natur ist, zeigt beispielsweise die Tatsache, dass die spanische Grossbank Santander seit Mitte April 2018 das Ripple-Protokoll für Transaktionen zwischen Spanien, Brasilien, Polen und Grossbritannien einsetzt. 36

\section{Zivilrechtlicher Teil}

\section{Ausgangslage}

17 Blockchain-Transaktionen erfolgen grenzüberschreitend, 37 womit auch international-privatrechtliche Implikationen zu berücksichtigen sind. Die nachfolgenden Ausführungen beziehen sich ausschliesslich auf Konstellationen, in denen aufgrund des massgebenden Kollisionsrechts das schweizerische Recht zur Anwendung gelangt, $3^{8}$ ein Szenario, dass namentlich durch den Umzug von bitfinex in die Schweiz vermehrt eintreten dürfte. Die Frage, was eine «Sache» ist, bzw. die Bestimmung des Umfangs des Sachbegriffs, ergibt sich aus den Bestimmungen des Sachstatuts, bei Anwendbarkeit des Schweizer Rechts also nach Massgabe des ZGB. 39

35 Patrick Griffin/Ryan Zagone, Assessing the Ripple Protocol: Implications of Distributed Networks and Digital Currencies for Retail Payments, 30. Januar 2015.

36 Santander launches the first blockchain-based international money transfer service across four countries, Press room, 12. April 2018.

37 Vgl. oben II.2.

38 Vgl. hierzu Barbara Graham-Siegenthaler/ Andreas Furrer, Jusletter, 8. Mai 2017, N 20 ff.

39 Pius Fisch, Vorbem. Art. 97-108, in: Honsell/Vogt/Schnyder/Berti (Hrsg.), Basler Kommentar, Internationales Privatrecht (IPRG), 3. Aufl., Basel 2013, N 9; Anton Heini, Zürcher Kommentar IPRG, 2. Aufl., Zürich 2004, Vorbem. Art. 97-108 N 11.
18 Nach schweizerischem Sachenrecht ist dem Inhaber eines Rechts an einer Sache (sog. dingliches Recht) diese so zugeteilt, dass alle anderen Rechtsgenossen diese Zuteilung zu respektieren haben. ${ }^{40}$ Die Möglichkeit, dingliche Rechte (insbesondere Eigentumsrechte) an Kryptowährungseinheiten begründen zu können, setzt somit voraus, dass Kryptowährungen als «Sachen» im Sinne des ZGB qualifiziert bzw. wie solche behandelt werden können. Die zurzeit vorherrschende Meinung zum deutschen Recht 41 wie wohl auch zum Schweizer Recht42

40 Arthur Meier-Hayoz, Berner Kommentar ZGB, Bern 1981, System. Teil, N 242; Jörg Schmid/Bettina Hürlimann-Kaup, Sachenrecht, 5. Aufl., Bern 2017, N 18.

$41 \S 90$ BGB sieht im Gegensatz zum ZGB die Voraussetzung der Körperlichkeit ausdrücklich vor, weshalb eine analoge Anwendung dieser Bestimmung weitgehend abgelehnt wird, vgl. etwa Franziska Boehm/Ina Bruns, in: Bräutigam/Rücker (Hrsg.), E-Commerce, München 2016, N 5; Merih Erdem Kütük/Christoph Sorge, Bitcoin im deutschen Vollstreckungsrecht: Von der «Tulpenmanie» zur «Bitcoinmanie», MMR 2014, S. 644; Albert Schlund/Hans Pongratz, DStR 2018, S. 600; Christian Engelhardt/Sascha Klein, Bitcoins - Geschäfte mit Geld, das keines ist: Technische Grundlagen und zivilrechtliche Betrachtung, MMR 2014, S. 357.

42 Aktuell diskutiert wird vorwiegend die Sachqualität von Daten bzw. «virtuellen Gütern» im Allgemeinen, wo sie aufgrund der fehlenden Körperlichkeit vom überwiegenden Teil der Lehre verneint wird, vgl. etwa Gianni Fröhlich-Bleuer, Jusletter, 6. März 2017 N 10; Daniel Hürlimann/Herbert Zech, Rechte an Daten, in: suigeneris 2016, S. 90 f.; Matthias Nänni, Märkte virtueller Welten, Zürich 2009, S. 133 ff.; anderer Ansicht demgegenüber Martin Eckert, Digitale Daten als Wirtschaftsgut: digitale Daten als Sache, SJZ 2016, S. 249. Ausdrücklich die Sachqualität von Kryptowährungen verneinend: Sébastien Gobat, Les monnaies virtuelles à l'épreuve de la LP, AJP 2016, S. 1098; François Piller, AJP 2017, Virtuelle Währungen - Reale Rechtsprobleme?, S. 1429; Harald Bärtschi/Christian Meisser, Virtuelle Währungen aus finanzmarkt- und zivilrechtlicher Sicht, in: Weber/Thouvenin (Hrsg.), Rechtliche Herausforderungen durch webbasierte und mobile Zahlungssysteme, Zürich 2015, 
verneint die Sachqualität von Kryptowährungen. In der Literatur wurden jüngst jedoch auch Stimmen laut, welche die Sachqualität in Bezug auf die grösste Kryptowährung Bitcoin bejahen. 43 Nachfolgend werden die geltend gemachten Argumente zusammengetragen und auch mit Blick auf andere Kryptowährungen gewürdigt.

\section{Der Sachbegriff des ZGB als dynamischer Begriff}

19 Das ZGB kennt keine Legaldefinition des Begriffs «Sache». Nach der herrschenden Auffassung stellt eine Sache ein unpersönlicher, körperlicher, für sich bestehender Gegenstand dar, welcher der menschlichen Herrschaft unterworfen werden kann.44 Daraus ergeben sich die Begriffsmerkmale Abgegrenztheit, Unpersönlichkeit, Körperlichkeit und Beherrschbarkeit.45 Methodologisch ist $\mathrm{zu}$ berücksichtigen, dass es sich beim Begriff «Sache» um einen Funktionsbegriff handelt. ${ }^{6}$ Der Sachbegriff ist somit nicht

S. 143. Frage offen gelassen bei Jacquemart/Meyer (Fn. 24), S. 469.

43 Graham-Siegenthaler/Furrer (Fn. 38), N 40 ff. Vgl. auch Eckert (Fn. 42), S. 247 ff. Teilweise abweichend Piller (Fn. 42), S. 1429, welcher die Qualifikation von Bitcoins als Sache jedoch als «wünschenswert» bezeichnet.

44 Heinz Rey, Die Grundlagen des Sachenrechts und des Eigentums, 3. Aufl., Bern 2007, N 66; MeierHayoz (Fn. 40), N 242; Thomas Sutter-Somm, Schweizerisches Privatrecht (SPR), Bd. V/1: Eigentum und Besitz, 2. Aufl., Basel 2014, N 16; Wolfgang Wiegand, Basler Kommentar ZGB II, 5 . Aufl., Basel 2015, Vorbem. zu Art. 641 ff. N 6; Paul-Henri Steinauer, Les droits réels, Tome I, 4. Aufl., Bern 2012, N 60; Ruth Arnet, in: Breitschmid/Jungo (Hrsg.), Handkommentar zum schweizerischen Privatrecht, Art. 641- 977 ZGB, 3. Aufl., Zürich 2016, Art. 641 N 6.

45 Statt vieler Sutter-Somm (Fn. 44), N 17 ff.

46 Meier-Hayoz (Fn. 40), N 116; Rey (Fn. 44), N 68; Wiegand (Fn. 44), Vorbem. zu Art. 641 ff. N 6. statisch sondern dynamisch, worauf zurückzukommen ist. 47

20 Wichtige Hilfsmittel der Gesetzesauslegung und -fortbildung sind die sog. Prinzipien des Sachenrechts, welche sich aus mehreren im Sachenrecht enthaltenen Rechtsnormen oder Rechtsinstituten durch Abstraktion ableiten lassen. Diese Prinzipien haben für das Sachenrecht eine besonders grosse Bedeutung, weil das ausdrückliche Bekenntnis des Gesetzgebers zur Unvollständigkeit und Lückenhaftigkeit i.S.v. Art. 1 ZGB (sog. «Mut zur Lücke») in besonderem Ausmass für das Sachenrecht gilt.48 Das wichtigste Prinzip stellt dabei das Publizitätsprinzip dar, welches besagt, dass dingliche Rechte als absolute Rechte für jedermann erkennbar sein sollen. Mit dem Publizitätsprinzip hängen eine Reihe weiterer allgemeiner Prinzipien des Sachenrechts zusammen. So namentlich das Traditions- und das Spezialitätsprinzip. Ein ebenfalls zentrales Prinzip stellt der Grundsatz dar, wonach das früher errichtete dingliche Recht dem später errichteten Recht vorgeht (sog. Prinzip der Alterspriorität).49

\section{Kryptowährungen als «Sache» im Sinne des ZGB?}

\section{a) Unpersönlichkeit}

21 Als Sache im Sinne des ZGB kann zunächst nur gelten, was vom menschlichen Körper verschieden ist. Das Vorliegen dieser Voraussetzung bereitet im Zusammenhang mit Kryptowährungen keinerlei Probleme und bedarf keiner weiteren Ausführungen.

47 Vgl. unten III.3.c.

48 Rey (Fn. 44), N 270.

49 Meier-Hayoz (Fn. 40), N 97; Rey (Fn. 44), N 550. 


\section{b) Abgegrenztheit}

Abgegrenztheit setzt voraus, dass die Sache für sich selber besteht und von anderen Sachen abgegrenzt werden kann..$^{\circ}$ Entsprechend der Funktionalität des Sachbegriffs ist auch das Begriffsmerkmal der Abgegrenztheit funktional aufzufassen. ${ }^{51}$ So erfüllt nicht nur eine physikalisch-räumlich abgegrenzte Sache (wie z.B. ein Buch) diese Voraussetzung. Ausschlaggebend kann auch die sich auf wirtschaftliche Momente abstützende Abgegrenztheit und Einheit sein (sog. wirtschaftliche Einheit). ${ }^{2}$ So kommt etwa bei einem Sack Getreide nicht dem einzelnen Getreidekorn die Sachqualität $\mathrm{zu}$, sondern der gesamten Menge, welche eine wirtschaftliche Einheit bildet und nur als solche am Rechtsverkehr teilnimmt.53 Diese Voraussetzung steht in engem Zusammenhang mit dem Spezialitätsprinzip, wonach dingliche Rechte stets an individualisierten (d.h. von anderen Sachen abgegrenzten) Objekten bestehen können.54 Letzteres ist seinerseits Ausfluss des Publizitätsprinzips, zumal das Spezialitätsprinzip verhindern soll, dass die Publizität des dinglichen Rechts durch Verfügungen, welche sich nicht auf eine individualisierte, abgegrenzte Objekte beziehen, verwischt wird. 55

In der Literatur wird zu Recht betont, dass eines der Hauptmerkmale von Bitcoin (BTC) die Transparenz ist, welche - wie aufgezeigt 56 - durch die öffentliche

\footnotetext{
Statt vieler Rey (Fn. 44), N 69.

Rey (Fn. 44), N 69.

Rey (Fn. 44), N 69; Steinauer (Fn. 44), N 64; Meier-Hayoz (Fn. 40), N 121 ff.

3 Sutter-Somm (Fn. 44), N 17.

Rey (Fn. 44), N 333.

Meier-Hayoz (Fn. 40), N 75; Rey (Fn. 44), N 336.

Vgl. oben II.1.
}

Blockchain gewährleistet wird, was auch auf Ethereum (ETH) und Ripple (XRP) zutrifft.57 Die Möglichkeit, die Transaktionskette beliebig weit zurückzuverfolgen, $5^{8}$ sowie die Irreversibilität jeder Übertragung59 schaffen ein hohes Mass an Sicherheit, dass der Empfänger dieser Kryptowährungseinheiten tatsächlich deren Berechtigter ist. Dieses Mass an Sicherheit übertrifft namentlich jenes, welches der Besitz bei Mobilien gewährleistet, bei weitem. ${ }^{60} \mathrm{Da}$ sich die Übertragung von Kryptowährungen stets auf eine bestimmte Anzahl von Einheiten bezieht, besteht Einklang mit dem Publizitäts- und dem Spezialitätsprinzip. ${ }^{61}$ Schliesslich ist zu berücksichtigen, dass jede einzelne Währungseinheit individuell bestimmbar ist und somit von anderen Währungseinheiten abgegrenzt werden kann. ${ }^{62}$ Aus einem funktionalen Blickwinkel erfüllen Kryptowährungen damit die Voraussetzung der Abgegrenztheit. Wie es sich mit der Notwendigkeit einer körperlichen Abgegrenztheit verhält, ergibt sich aus der nachfolgend zu prüfenden Notwendigkeit der Körperlichkeit.

24 Vorab ist mit Blick auf das Altersprioritätsprinzip festzustellen, dass Kryptowährungen aufgrund der komplexen technischen Lösung zur Verhinderung des sog. double-spend-Problems, d.h. Verunmöglichung der mehrfachen Über-

\footnotetext{
57 Vgl. oben II.3 und II.4.

58 Vgl. oben II.2.

59 Vgl. oben II.2 (BTC), II.3 (ETH) und II.4 (XRP).

6o Zur Problematik der Abgrenzung zwischen «verdächtigem» und «unverdächtigem» Besitz vgl. etwa Urteil des Bundesgerichts 5A_521/2008 vom 5. Januar 2009 E. 4 f.; Wolfgang Ernst, Basler Kommentar ZGB II, 5. Aufl., Basel 2015, Art. $930 \mathrm{~N} 7 \mathrm{ff}$.

61 Graham-Siegenthaler/Furrer (Fn. 38), N 43.

62 Vgl. oben II.1.
} 
tragung von Kryptowährungseinheiten, ${ }^{63}$ auch gewährleistet ist, dass der früher übertragenen Berechtigung der Vorrang vor der späteren zukommt. 64

\section{c) Körperlichkeit}

Das Erfordernis der Körperlichkeit ist erfüllt, wenn es sich um einen greifbaren Gegenstand handelt.65 Kryptowährungen fehlt diese Eigenschaft offensichtlich. Hierin liegt der Grund, weshalb diesen die Sachqualität von der überwiegenden Lehre bislang abgesprochen wird. ${ }^{66} \mathrm{Zu}$ beachten ist allerdings, dass diese Einschränkung auf körperliche Gegenstände im ZGB nicht durchgehend eingehalten wird. In gewissen Fällen stellt das Gesetz unkörperliche «Objekte» den Sachen gleich. So erklärt insbesondere Art. 713 ZGB Naturkräfte (und somit auch Energie $^{67}$ ) zum Gegenstand von Fahrniseigentum, womit diese im Bereich des Eigentumsrechts wie (bewegliche) Sachen behandelt werden. 68 Voraussetzung für die Behandlung als bewegliche Sache ist, dass die Naturkräfte der rechtlichen Herrschaft unterworfen werden können.

Der Umstand, dass das Gesetz gewisse unkörperliche Objekte ausdrücklich wie Sachen behandelt, lässt Zweifel aufkom-

63 Vgl. oben II.2.

64 Vgl. auch Graham-Siegenthaler/Furrer (Fn. 38), $\mathrm{N} 43$.

65 Sutter-Somm (Fn. 44), N 18; Schmid/HürlimannKaup (Fn. 40), N 7; Rey (Fn. 44), N 81; Wiegand (Fn. 44), Vorbem. zu Art. 641 ff. N 10.

66 Vgl. die Hinweise in Fn. 42.

67 Vgl. bereits BGE 48 II 366 E. 2.

68 Daneben bestehen weitere Ausnahmen von der Körperlichkeit: Nach Art. 655 Abs. 2 Ziff. 2 ZGB werden bestimmte Rechte wie unbewegliche Sachen behandelt. Aus Art. 745 und Art. 899 ff. ZGB ergibt sich, dass beschränkte dingliche Rechte nicht nur an körperlichen Sachen, sondern auch an Forderungen und anderen Rechten bestehen können. men, ob das durch Lehre und Rechtsprechung hergeleitete Element der Körperlichkeit ausnahmslos als strikte Voraussetzung der Behandlung eines Objektes als Sache angesehen werden kann. So führte bereits Meier-Hayoz unter Verweis auf Wieacker 69 aus, dass die Qualifikation eines Objektes als Sache «nicht alleine von der physikalischen Beschaffenheit» abhänge, «sondern vor allem von der wirtschaftlichen Funktion, von der Verkehrsanschauung und von ethischen Gesichtspunkten», weshalb teleologische Erwägungen den Ausschlag geben sollten. ${ }^{70}$ Dieser Aussage haben sich diverse Autoren angeschlossen. ${ }^{71}$ Wiegand kommt zum Schluss, dass die funktionale Ausrichtung des Sachbegriffs eine Qualifizierung «elektronischer Gegenstände» als Sachen grundsätzlich zulasse, wodurch sich Modifikationen des tradierten Sachbegriffs aufdrängen könnten. Diese rechtspolitische Frage sei demnächst in einheitlicher Weise zu entscheiden, wolle man elektronische Ver-

$69 \overline{\text { Franz Wieacker, Sachbegriff, Sacheinheit und }}$ Sachzuordnung, AcP 1943, S. 73 ff., vgl. insbesondere S. 74: «Die Frage der rechtlichen Sachqualität hat nicht die Einheit des natürlichen Zusammenhangs oder unserer sinnlichen Wahrnehmungen zum Gegenstand, sondern die richtige Ordnung der Sachgüterverteilung; sie fragt nicht danach, was eine Sache ist, sondern was gerechterweise als einheitlicher Gegenstand nur einem Vermögen und einer Rechtslage zugeordnet werden kann. Nicht die natürliche Körperwelt als solche, sondern ihre sozialen, besonders ihre wirtschaftlichen oder kulturellen Wertgehalte sind ja der Gegenstand des Vermögensrechts».

70 Meier-Hayoz (Fn. 40), N 116. Vgl. auch Robert Haab/Pascal Simonius, Zürcher Kommentar, Bd. IV/1: Das Eigentum, 2. Aufl., Zürich 1977, Einleitung vor Art. $641 \mathrm{~N} 40$.

71 Vgl. etwa Arnet (Fn. 43), Art. 641 N 6; Rey (Fn. 44), N 68; Stephanie Hrubesch-Millauer/ Barbara Graham-Siegenthaler/Vito Roberto, Sachenrecht, Bern 2017, N 01.06 f. 
kehrsvorgänge nicht weiterhin unnötig behindern..$^{2}$

Vor diesem Hintergrund sprechen beachtliche Gründe dafür, das Element der Körperlichkeit im Sinne einer zeitgemässen Auslegung unter gewissen Umständen nicht als strikte Voraussetzung für die Behandlung eines Gegenstands als Sache im Sinne des ZGB oder zumindest als Gegenstand des Eigentums aufzufassen. Bei der Entscheidung der Frage, ob ein konkreter unkörperlicher Gegenstand wie eine Sache behandelt werden kann, muss der Voraussetzung der Beherrschbarkeit jedoch besondere Bedeutung zukommen.73

\section{d) Beherrschbarkeit}

Beherrschbarkeit setzt die Möglichkeit voraus, den fraglichen Gegenstand tatsächlich und rechtlich zu beherrschen, sei dies durch Erwerb oder Nutzung.74 Tatsächliche Beherrschbarkeit fehlt bei Gegenständen, die für den Menschen aufgrund fehlender technischer Möglichkeiten nicht beherrschbar sind. 75 Im Be-

${ }_{72}$ Wiegand (Fn. 44), Vorbem. zu Art. 641 ff. N 6.

73 Dies ergibt sich einerseits daraus, dass dingliche Rechte unmittelbar die tatsächliche Herrschaft über die betreffende Sache vermitteln (vgl. statt vieler Rey [Fn. 44], N 575), was sich vorwiegend auf körperlicher Ebene manifestiert und folglich bei Ausweitung des Sachbegriffs auf Unkörperliches einer besonderen Prüfung bedarf. Dementsprechend hat der Gesetzgeber bei Naturkräften nicht ersatzlos auf die Voraussetzung der Körperlichkeit verzichtet, sondern diese gewissermassen durch eine besondere Betonung der Voraussetzung der Beherrschbarkeit ersetzt (vgl. Art. 713 Halbsatz 2 ZGB).

74 Statt vieler Sutter-Somm (Fn. 44), N 19.

75 Die Voraussetzung der rechtlichen Beherrschbarkeit schliesst die Sachqualität beispielswiese am menschlichen Körper oder Objekten der Fortpflanzungsmedizin aus, vgl. statt vieler SutterSomm (Fn. 44), N 20. Diese Voraussetzung bietet im Zusammenhang mit Kryptowährungen keinerlei Probleme. reich der digitalen Güter ist die Beherrschbarkeit technisch grundsätzlich ohne weiteres gegeben. Dies trifft auch auf Kryptowährungen zu. 76

29 Gegen die Sachqualität von Daten wird jedoch vorgebracht, dass das Eigentumsrecht als Ausschliesslichkeitsrecht nur rivalisierende Nutzungen 77 erfassen kann. ${ }^{78}$ Daten fehlt diese Eigenschaft, zumal solche von mehreren Personen gleichzeitig verwendet werden können, ohne dass eine Verwendung die andere beeinträchtigt (nicht-rivalisierende Nutzung).79 In der Tat ist es sinnlos, ein Objekt als Sache im Rechtssinne zu behandeln, wenn daran kein Eigentum begründet werden kann, da eine ausschliessliche Zuweisung des entsprechenden Objekts nicht möglich ist. Hier ist jedoch auf eine wesentliche Besonderheit der Kryptowährungen zurückzukommen: Der Erwerber einer Kryptowährungseinheit erwirbt, gleich wie beim Erwerb von Bargeld, die ausschliessliche faktische Möglichkeit, dieselbe auf einen neuen Erwerber zu übertragen. Eine entsprechende Transaktion mittels Blockchain ist ohne aktive Beteiligung des Inhabers (bzw. Verwendung des private keys) technisch ausgeschlossen. ${ }^{80}$ Die Weitergabe des private

76 Gl. M. Graham-Siegenthaler/Furrer (Fn. 38), N 67; Meyer/Schuppli (Fn. 23), S. 220.

77 Körperliche Güter können nur von einer bestimmten Person genutzt werden (rivalisierende Nutzung), während geistige Güter (z.B. ein $\mathrm{Mu}-$ sikstück) gleichzeitig von einer unbeschränkten Vielzahl von Personen genutzt werden können, ohne dass die Nutzung durch eine Person diejenige durch die anderen beeinträchtigen würde (nicht-rivalisierende Nutzung).

78 Hürlimann/Zech (Fn. 42), N 8. Vgl. auch Florent Thouvenin, Urheberrecht statt «geistiges Eigentum», NZZ Nr. 193 vom 21. August 2012, S. 19.

79 Hürlimann/Zech (Fn. 42), N 2 und 8.

80 Vgl. oben II.2. 
keys führt aufgrund der Verunmöglichung des double-spending ${ }^{81}$ nicht zu einer gleichzeitigen Nutzungsmöglichkeit verschiedener Personen. Ebenfalls technisch ausgeschlossen ist das Kopieren bzw. Multiplizieren einer Kryptowährungseinheit. ${ }^{82}$ Der Inhaber ist folglich in Bezug auf die Nutzung von Kryptowährungen, welche ausschliesslich in der Übertragung der Berechtigung zur weiteren Übertragung liegt, ${ }^{83}$ mit ausschliesslicher Herrschaft ausgestattet und zwar selbst dann, wenn der Inhaber für die Aufbewahrung und Transaktionen die Dienstleistungen eines BlockchainAnbieters in Anspruch nimmt. ${ }^{84}$ In diesem Sinne ist bei Kryptowährungen im Gegensatz zu Daten eine nichtrivalisierende Nutzung ausgeschlossen. Darin liegt gewissermassen das Wesen der zugrundeliegenden Blockchain bzw. Distributed-Ledger-Technologie (DLT).

\section{e) Weitere Gesichtspunkte}

3o Kryptowährungen werden wie Geld bzw. Tauschmittel wahrgenommen und behandelt, indem die Übertragung derselben zunehmend auch als Gegenleistung für den Erwerb von Sachen oder Dienstleistungen verwendet wird. ${ }^{85}$ Die wirtschaftliche Funktion bzw. die Verkehrsanschauung spricht folglich für eine Behandlung von Kryptowährungen als bewegliche Sachen.

$31 \mathrm{Zu}$ berücksichtigen ist sodann, dass die Behandlung von Kryptowährungen als Sachen und damit als möglichen Gegenstand des Fahrniseigentums eine relativ

1 Vgl. oben II.2.

2 Vgl. oben II.2.

83 Vgl. Kütük/Sorge (Fn. 41), S. 643.

84 Graham-Siegenthaler/Furrer (Fn. 38), N 33 und 38.

85 Vgl. Piller (Fn. 42), S. 1429; Schlund/Pongratz (Fn. 41), S. 600. klare zivilrechtliche Erfassung ermöglicht und damit einhergehend eine (analoge) Anwendung von bewährten Regeln zur Folge hat, was im Sinne der Förderung der für den Wirtschaftsverkehr wichtigen Rechtssicherheit auf dem Gebiet der Kryptowährungen ist. ${ }^{86}$ Mit der Anerkennung von Eigentum an Kryptowährungseinheiten folgt beispielsweise die Möglichkeit zur Aussonderung derselben im Konkurs (Art. 242 SchKG), was als sachgerecht erscheint und den Erwartungen der Rechtssuchenden entspricht. ${ }^{87}$ Ein anschauliches Beispiel hierfür stellt der Konkurs der in Japan domizilierten und ehemals weltweit grössten Handelsplattform «Mt. Gox» im Jahre 2014 dar, welcher bis heute noch für eine grosse Verunsicherung unter Investoren sorgt. 88

32 Als weitere Konsequenz stünde dem Inhaber die Eigentumsklage (Art. 641 Abs. 2 ZGB) offen, wenn die Übertragung von Kryptowährungen auf einem ungültigen Rechtsgrund beruht und das Eigentum somit mangels gültigen Grundgeschäfts nicht übergegangen ist oder wenn dem Inhaber die Herrschaft an seinen Kryptowährungseinheiten abhandengekommen ist (namentlich durch Diebstahl bzw. unberechtigte Verwendung des private keys). 89 Vorausgesetzt ist hierbei, dass durch die erfolgte Übertragung kein Eigentumsübergang auf den

86 Vgl. auch Piller (Fn. 42), S. 1429.

87 Eingehend hierzu Graham-Siegenthaler/Furrer (Fn. 38), N 93 ff. Vgl. auch Piller (Fn. 42), S. 1437.

88 Vgl. hierzu etwa: Robin Sidel/Eleanor Warnock/Takashi Mochizuki, Almost Half a Billion Worth of Bitcoins Vanish: Mt. Gox Says It Lost 750,000 of Customers' Bitcoin to Fraud, The Wall Street Journal Online, 28. Februar 2014.

89 Zur Vindikation von Kryptowährungseinheiten vgl. auch Meyer/Schuppli (Fn. 23), S. 219 f. 
Empfänger erfolgt und die Aktivlegitimation des Klägers somit entfallen ist. Im Zusammenhang mit Geld ist der Anwendungsbereich der Eigentumsklage durch die Möglichkeit des originären Eigentumserwerbs auf Seiten des neuen Besitzers durch Vermischung stark eingeschränkt.90 Eine solche Vermischung setzt voraus, dass die einzelnen Währungseinheiten nicht mehr voneinander unterschieden werden können. Bei Kryptowährungen ist die Situation jedoch so, dass jede einzelne Währungseinheit individuell bestimmbar ist, da sie auf einer individuellen in der Signaturkette abgebildeten Transaktion beruht. ${ }^{91}$ Ein originärer Eigentumserwerb zufolge Vermischung ist somit - jedenfalls bei pseudonymen Kryptowährungen wie Bitcoin (BTC), Ethereum (ETH) oder Ripple (XRP) - grundsätzlich ausgeschlossen. ${ }^{22}$ Für den Fall der Weiterübertragung durch den (unberechtigten) neuen Inhaber auf eine gutgläubige Drittperson wäre zu prüfen, ob durch analoge Anwendung von Art. 935 ZGB wie bei Geld und Inhaberpapieren für eine Berücksichtigung der Verkehrsinteressen gesorgt werden müsste, was aufgrund der steten Zunahme der Akzeptanz von Kryptowährungen als Zahlungsmittel als angezeigt erscheint.

\footnotetext{
Vgl. etwa BGE 136 III 247 E. 5 .

Vgl. oben II.2.

92 Meyer/Schuppli (Fn. 23), S. 220. Bei anonymen Kryptowährungen wie NEM, Monero, Dash oder ZCash wäre die Möglichkeit der Vermischung demgegenüber wohl gegeben. Als Folge eines Diebstahls einer grossen Menge an NEM auf der japanischen Tauschbörse Coincheck im Januar 2018 und dem Umstand, dass nur ein Teil der entwendeten Einheiten identifiziert und gesichert werden konnte, kündigte Coincheck Mitte März 2018 die Einstellung des Handels mit NEM, Monero, Dash und ZCash an.
}

33 Die Herausgabepflicht würde durch die Rückübertragung bzw. die Herausgabe des private keys durch den Verpflichteten vollzogen und wäre auf dem Weg der Realvollstreckung (Art. 335 ff. ZPO) durchzusetzen. Ist eine Herausgabe aus technischen Gründen nicht möglich, z.B. weil der private key nicht mehr auffindbar ist oder die Einheiten bereits in eine die Herausgabe verunmöglichenden Weise weiterübertragen wurden, verbliebe dem bisherigen Inhaber ein Schadenersatzanspruch gestützt auf Art. 41 OR bzw. ein Ersatzanspruch gestützt auf Art. 62 ff. OR.93 Dies könnte aufgrund der hohen Volatilität von Kryptowährungen und der für die Durchsetzung des Schadenersatzanspruchs notwendigen Zeit problematisch und mit wirtschaftlichen Nachteilen verbunden sein. Doch auch in dieser Situation kann auf Regeln zurückgegriffen werden, welche die Lehre und Rechtsprechung im Zusammenhang mit Wertveränderungen von Aktien entwickelt hat.94

$93 \overline{\text { Konnte die Unmöglichkeit der Realvollstreckung }}$ nicht im Erkenntnisverfahren berücksichtigt werden, kann der obsiegende Kläger gestützt auf Art. 345 Abs. 1 lit. a ZPO Schadenersatz verlangen. In diesem Fall kann als Schaden der Wert der untergegangenen Primärleistung zum Zeitpunkt des Urteils zuzüglich Verzugszins verlangt werden (Erfüllungsinteresse), weiterer Schaden (indirekter Schaden oder über den Verzugszins hinausgehender Wertzuwachs) kann in einem neuen Erkenntnisverfahren geltend gemacht werden, vgl. Daniel Staehelin, in: SutterSomm/Hasenböhler/Leuenbeger (Hrsg.), ZPO Kommentar, 3. Aufl., Zürich 2016, Art. 345 N 5.

94 Insbesondere soll der i.S.v. Art. 41 OR Geschädigte grundsätzlich die Wahl haben, welcher Zeitpunkt für die Schätzung der Schadenshöhe massgebend sein soll. Ist der Kurs zwischen dem Schädigungs- und dem Urteilstag gesunken, so gilt dieser Faktor gegenüber dem Schadensentstehungstag als hypothetische Kausalität, was zur Massgeblichkeit dieses Zeitpunkts führt. Wenn umgekehrt der Kurs zwischen dem Schädigungsund dem Urteilstag gestiegen ist, so hat sich der Schaden vergrössert, weil zwischenzeitlich der 
34 In der Praxis häufig anzutreffende Verträge zwischen Inhaber und einem Dienstleister, welche die Fremdverwaltung von private keys zum Gegenstand haben (sog. Wallet-Provider), wären sodann als Hinterlegungsverträge gemäss Art. 472 ff. OR zu qualifizieren.95 Der Auftrag zur Beschaffung von Kryptowährungseinheiten stellte ein Kommissionsgeschäft nach Art. 425 OR dar. ${ }^{96}$

35 Wird demgegenüber die Sachqualifikation von Kryptowährungen abgelehnt und damit die Begründung von Eigentum ausgeschlossen, erweist sich die rechtliche Zuordnung von Kryptowährungen zum Inhaber als schwierig. Kryptowährungen sind weder Immaterialgüter noch Persönlichkeitsgüter.97 Ein absoluter Schutz durch Verknüpfung mit dem absoluten Recht am Datenträger ist aufgrund der dezentralen Struktur der Blockchain bzw. der Distributed-Ledger-

Geschädigte noch nicht befriedigt wurde. Vgl. hierzu Roland Brehm, Berner Kommentar OR, Bern 2005, Art. 42 N 8 mit Verweis auf Andreas von Tuhr/Hans Peter, Allgemeiner Teil des schweizerischen Obligationenrechts, Bd. 1, Zürich 1979, S. 123 und BGE 109 II 474. Im Übrigen ermöglichen Art. 43 Abs. 1 und Art. 44 Abs. 1 OR eine angemessene Berücksichtigung der konkreten Umstände des Einzelfalls bei der Bestimmung des Umfangs der Ersatzpflicht. Zum deutschen Recht in diesem Zusammenhang vgl. Engelhardt/Klein (Fn. 41), S. 358.

95 Jacquemart/Meyer (Fn. 24), S. 478 mit dem Hinweis darauf, dass der Hinterleger im Falle eines sog. Hardforks (eingehend hierzu S. $468 \mathrm{ff}$. hinsichtlich des prominentesten Beispiels Bitcoin Cash) die neu angefallenen Kryptowährungseinheiten gemäss Art. 475 Abs. 1 OR herausgefordert werden könnten.

96 Jacquemart/Meyer (Fn. 24), S. 478.

97 Piller (Fn. 42), S. 1428; Franziska Boehm/Paulina Pesch, Bitcoins: Rechtliche Herausforderungen einer virtuellen Währung - Eine erste juristische Einordung, MMR 2014, S. 78, Engelhardt/Klein (Fn. 41), S. 357; Boehm/Bruns (Fn. 41), N 7 f.; Schlund/Pongratz (Fn. 41), S. 600
Technologie (DLT) ausgeschlossen.98 Ebenfalls können Kryptowährungen mangels einer zentralen emittierenden Stelle als solche nicht als Forderung qualifiziert werden.99 Zwar wären Verträge, welche Kryptowährungen zum Gegenstand haben, aufgrund der Vertragsfreiheit grundsätzlich wirksam. Doch würde bereits die Qualifikation derartiger Verträge Schwierigkeiten bereiten ${ }^{100}$ und kaum zu befriedigenden Ergebnissen führen. ${ }^{101}$ Unklarheiten bestünden desgleichen hinsichtlich der Zwangsvollstreckung. ${ }^{102}$

\section{Fazit}

36 Aus zivilrechtlicher Sicht ist es wünschenswert, Kryptowährungen als Sachen zu behandeln bzw. diese zumindest mit einer absoluten Schutzwirkung auszustatten. ${ }^{103}$ Eine klare Regelung durch den Gesetzgeber - sei es durch Schaffung einer Art. 713 ZGB entsprechenden Regelung oder durch Normierung bestimmter absoluter Rechtswirkungen wie bei den sog. Bucheffekten ${ }^{104}$ - erscheint als angezeigt und stellt u.E. eine Chance für die Wettbewerbs- und Zukunftsfähigkeit des Finanzplatzes Schweiz dar.

$98 \overline{\text { Vgl. Boehm/Bruns (Fn. 41), N 5; Schlund/ }}$ Pongratz (Fn. 41), S. 600. Abweichend offenbar Engelhardt/Klein (Fn. 41), S. 357.

99 Boehm/Bruns (Fn. 41), N 7; Boehm/Pesch (Fn. 97), S. 78; Schlund/Pongratz (Fn. 41), S. 60.

100 Vgl. Jacquemart/Meyer (Fn. 24), S. 478 hinsichtlich der erwähnten Verträge, welche die Fremdverwaltung von private keys bzw. die Beschaffung von Kryptowährungseinheiten beinhalten.

101 Vgl. Boehm/Pesch (Fn. 97), S. 77 f., Boehm/Bruns (Fn. 41), N 7 und N 12 ff.; Engelhardt/Klein (Fn. 41), S. 359 f.; Schlund/Pongratz (Fn. 41), S. $600 \mathrm{f}$.

${ }_{102}$ Schmid/Schmid, N 27 ff. Vgl. auch Kütük/Sorge (Fn. 41), S. 644 f.; Boehm/Bruns (Fn. 41), N 43 ff.

103 Gl. M. Piller (Fn. 42), S. 1428; GrahamSiegenthaler/Furrer (Fn. 38), N 72 ff. Vgl. auch Engelhardt/Klein (Fn. 41), S. 359 f.

104 Vgl. insbesondere Art. 29 Abs. 3 BEG. 
37 Die Ausführungen haben sodann gezeigt, dass der Ansicht beizupflichten ist, wonach Kryptowährungen bereits de lege lata im Sinne einer modernen Auslegung des Sachbegriffs wie eine Sache behandelt und damit als Gegenstand des Fahrniseigentums aufgefasst werden können. Letztlich wird aufgrund der grenzüberschreitenden Struktur von Kryptowährungen auch in zivilrechtlicher Hinsicht eine länderübergreifend abgestimmte Regelung notwendig sein. Aufgrund der Tatsache, dass die tatsächliche und wirtschaftliche Bedeutung von Kryptowährungen rasant steigt und derart grundlegende Gesetzgebungsarbeiten einige Zeit beanspruchen, werden sich Gerichte und Verwaltungsbehörden potentiell mit der Notwendigkeit der Rechtsfortbildung konfrontiert sehen, womit aber auch die einmalige Gelegenheit besteht, wichtige Pionierarbeit in dieser Hinsicht zu leisten. Hierzu möge dieser Aufsatz einen konkreten Denkanstoss bieten. 